\title{
Perfil sociodemográfico e clínico de pessoas com deficiência intelectual em um
}

\section{município de pequeno porte}

\author{
Sociodemographical and clinical profille of intellectual disabled people in a small \\ Perfil sociodemográfico y clínico de personas con discapacidad intelectual en un municipio pequeño
}

\section{Resumo}

A deficiência intelectual caracteriza-se como um transtorno do desenvolvimento que inclui prejuízos intelectuais e adaptativos, nos domínios conceitual, social e prático. Objetivou-se conhecer o perfil sociodemográfico e clínico de pessoas com deficiência intelectual de um município de pequeno porte do Rio Grande do Sul. Estudo exploratório, descritivo, com abordagem quantitativa, realizado entre os meses de abril a setembro de 2015, a partir da aplicação de um instrumento previamente elaborado para a coleta de dados. Foram encontradas 77 pessoas com deficiência intelectual com idades entre seis e 74 anos. A maioria eram do sexo masculino $(59,74 \%)$, solteiros (92,2\%) e analfabetos $(55,84 \%)$. Quanto ao diagnóstico clínico, destaca-se que 40,25\% dos sujeitos, conforme o CID-10, eram diagnosticados como F79 (Retardo Mental não especificado), sendo que em 50,64\% quem forneceu o diagnóstico foi o médico neurologista. $\mathrm{O}$ estudo revelou que a maioria das pessoas com deficiência intelectual estão na fase adulta e são solteiras; suas condições de vida/saúde são satisfatórias. Apesar de pertencentes a um município de pequeno porte, tais dados poderão colaborar com os profissionais e gestores de Educação e de Saúde, subsidiando assim discussões voltadas para o aprimoramento do cuidado longitudinal necessário junto a pessoas com deficiência intelectual.

Palavras-chave: Condições de vida; Condições de saúde; Deficiência intelectual; Qualidade de vida.

\begin{abstract}
Intellectual disability (ID) is characterized as a developmental disorder that includes intellectual and adaptive impairments in the conceptual, social and practical domains. Identify the sociodemographic and clinical profile of people with intellectual disabilities in a small city in the interior of Rio Grande do Sul (RS). A census and descriptive study, with a quantitative approach, carried between April and September 2015, through the application of a previously prepared instrument for data collection. There were 77 people with intellectual disabilities aged between six and 74 years. The majority were male (59.74\%), unmarried (92.2\%) and illiterate (55.84\%). Regarding the diagnosis, $40.25 \%$ of the subjects, according to ICD-10, were diagnosed as F79 (Unspecified Mental Retardation), and in $50.64 \%$ the diagnosis was given by the neurologist. The study found that most people with intellectual disabilities are in adulthood and are single; their living / health conditions are satisfactory. Although they belong to a small municipality, such data may collaborate with professionals and managers of Health and Education, thus subsidizing discussions aimed at improving the necessary longitudinal care among people with intellectual disabilities.
\end{abstract}

Keywords: Life conditions; Health conditions; Intellectual disability; Quality of life.

\section{Resumen}

La discapacidad intelectual se caracteriza como un trastorno del desarrollo que incluye impedimentos intelectuales y adaptativos, en los dominios conceptual, social y práctico. Conocer el perfil sociodemográfico y clínico de las personas con discapacidad intelectual en una pequeña ciudad del interior de Rio Grande do Sul. Censo y estudio descriptivo, con enfoque cuantitativo, realizado entre abril y septiembre de 2015, por mediante la aplicación de un instrumento previamente preparado para la recolección de datos. Se encontraron 77 personas con discapacidad intelectual con edades comprendidas entre los seis y los 74 años. La mayoría eran hombres (59,74\%), solteros (92,2\%) y analfabetos (55,84\%). En cuanto al diagnóstico, llama la atención que el 40,25\% de los sujetos, según la CIE-10, 
fueron diagnosticados como F79 (Retraso mental no especificado), y en el 50,64\% que brindó el diagnóstico fue el neurólogo. El estudio reveló que la mayoría de las personas con discapacidad intelectual están en la edad adulta y son solteras; sus condiciones de vida / salud son satisfactorias. A pesar de pertenecer a un municipio pequeño, estos datos pueden colaborar con los profesionales y gestores de Educación y Salud, apoyando así discusiones encaminadas a mejorar la atención longitudinal necesaria para las personas con discapacidad intelectual.

Palabras clave: Condiciones de vida; Condiciones de salud; Discapacidad intelectual; Calidad de vida.

\section{Introdução}

A deficiência intelectual (DI) é um transtorno do desenvolvimento que inclui prejuízos intelectuais e adaptativos, nos domínios conceitual, social e prático. Seu diagnóstico implica déficits em funções intelectuais como raciocínio, resolução de problemas, planejamento, pensamento abstrato, julgamento, aprendizagem acadêmica e pela experiência; e déficits em funções adaptativas que resultam em fracasso para atingir padrões de desenvolvimento e socioculturais em relação à independência pessoal e responsabilidade social (Apa, 2014).

Atualmente, o modelo da inclusão vem influenciando as políticas de atenção às pessoas com deficiência (Mantoan, 2015). De acordo com este paradigma, a deficiência não está instalada no indivíduo, cabendo somente a ele buscar o seu desenvolvimento, mas a sociedade também possui o papel de encontrar meios que proporcionem o desenvolvimento e a inclusão das pessoas com deficiência, tendo em vista o direito destas ao acesso a bens comuns da vida em comunidade (Mantoan, 2015).

A DI é um diagnóstico clínico presumível a partir dos cinco anos de idade, quando é possível mensurar adequadamente os déficits intelectuais e adaptativos (Apa, 2014). Essa deficiência tem prevalência global estimada entre $1 \mathrm{e}$ $3 \%$ e está presente como comorbidades em mais de 2.000 condições nosológicas distintas, incluindo muitas doenças genéticas raras (Schalock, 2010).

Dados do Censo Demográfico de 2010 indicam que 1,4\% da população brasileira possui algum grau de DI. No Rio Grande do Sul, estado onde se localiza o município estudado, encontra-se a proporção de 0,7\% de pessoas com DI (Ibge, 2010).

No Brasil, nas últimas décadas, especialmente, a partir Constituição Federativa de 1988 (Brasil, 1988), observa-se maior preocupação e interesse com essa parcela da população; destacam-se o direito à educação, saúde, lazer e trabalho. Nessa perspectiva, houve uma evolução na construção e consolidação de políticas públicas voltadas a garantir os direitos básicos às pessoas com DI. Entretanto, embora tenham ocorrido avanços, ainda há larga distância entre o previsto legalmente e a realidade vivenciada pelas pessoas com DI (Kassar, 2012).

Assim, o cuidado adequado a pessoa com DI torna-se como um debate importante a ser instigado entre profissionais de Educação e de Saúde. Há uma carência de informações a respeito das condições de saúde da pessoa com DI, as quais acabam favorecendo a continuidade de uma série de problemas sociais (Girardi et al, 2012). Estudos em relação à identificação e às características de saúde-doença das pessoas com DI são importantes tanto para o avanço nessa área do conhecimento, quanto para a produção de evidências para melhores práticas de cuidado (O’leary et al, 2018).

Considerando os aspectos acima referidos, perguntou-se: quantos são e como estão vivendo as pessoas com DI de um município de pequeno porte do interior do gaúcho? As políticas públicas relacionadas às pessoas com DI estão sendo devidamente aplicadas no município? Nesse sentido, o objetivo deste estudo foi conhecer o perfil sociodemográfico e clínico de pessoas com DI de um município de pequeno porte do interior do Rio Grande do Sul (RS), com aproximadamente 16 mil habitantes. 


\section{Metodologia}

Trata-se de um estudo de caráter exploratório, descritivo, com abordagem quantitativa (Pereira et al. 2018), realizada em um município de pequeno porte situado no interior do estado do RS. A população foi estabelecida por busca ativa nos estabelecimentos de saúde, de educação e de assistência social do município (Secretaria Municipal de Saúde, Secretaria Municipal e Estadual de Saúde, Associação de Pais e Amigos dos Excepcionais (APAE), Centro de Atenção Psicossocial I (CAPS I) e Instituição de Longa Permanência para Idosos [ILPI]).

Os critérios de inclusão adotados neste estudo foram: pessoas com DI com idade a partir de seis anos, com cuidador ou responsável, com idade superior a 18 anos. Os critérios de exclusão foram: famílias nas quais não foi possível identificar o cuidador principal e os sujeitos que não aceitaram participar da pesquisa.

O estudo foi realizado com 77 pessoas com DI, com idades entre seis e 74 anos, de ambos os sexos, sendo entrevistados os sujeitos com DI ou seus responsáveis, que foram devidamente esclarecidos e orientados sobre a natureza, o objetivo, do caráter estritamente voluntário e não invasivo do estudo e convidados a participar da pesquisa. Após a leitura e o entendimento do Termo de Consentimento Livre e Esclarecido (TCLE), os participantes assinavam, em duas vias, sendo-lhes entregue uma cópia. Nesse sentido, foram asseguradas a confidencialidade, a privacidade e a proteção da identidade dos sujeitos, buscando acima de tudo evitar riscos potenciais. Foram garantidos aos participantes a possibilidade de desistência em qualquer fase da pesquisa, sem ônus ou comprometimento de atendimento institucional.

A coleta de dados foi realizada por uma das autoras/pesquisadoras, nas residências dos sujeitos ou nas instituições, entre os meses de abril a setembro de 2015, por meio da aplicação de um instrumento previamente elaborado para a coleta de dados. As variáveis estudadas incluíram características sociodemográficas e econômica (sexo, idade, estado civil, convivência familiar, escolaridade, local e apropriação de moradia, renda familiar mensal e participação na vida econômica familiar), diagnóstico e acompanhamento clínico/terapêutico/educacional (profissional que forneceu o diagnóstico e acompanhamento clínico, tipo de acompanhamento clínico terapêutico e educacional) e a caracterização atual da saúde (visão, audição, presença de polifarmácia, principais medicações utilizadoas e hábitos nocivos à saúde [fumo e álcool]). Destaca-se que o instrumento de coleta de dados foi elaborado diante da percepção da necessidade de dados relevantes a este estudo, fundamentado na literatura nacional e internacional, na experiência e no contato profissional e acadêmico com a população estudada. Era composto por perguntas fechadas, de fácil aplicação e análise. O tempo médio gasto nas entrevistas foi de 50 minutos, que tiveram durações variadas, já que não foram colocados limites de tempo.

Ressalta-se que foram preservados os nomes e demais informações pessoais; para se garantir o anonimato dos sujeitos, foram utilizados letras e números (PDI1; PDI2; PDI3 - Pessoa com Deficiência Intelectual 1 e, assim, por diante).

Para a organização do banco de dados, foi utilizado o programa de computador Microsoft Excel, versão 2013.

O estudo foi aprovado pelo Comitê de Ética e Pesquisa (CEP) da Universidade onde foi realizado, sob parecer $\mathrm{n}^{\circ}$ 979.759, em 10 de março de 2015, seguindo, pois, a Resolução 466/12 do Conselho Nacional de Saúde (Brasil, 2012).

\section{Resultados}

A busca ativa realizada nos equipamentos de Saúde, Educação e Assistência Social do município resultou em 77 pessoas com DI (0,48\% da população total). Na Tabela 1 encontram-se a caracterização sociodemográfica e econômica dos sujeitos participantes da pesquisa, destacando as variáveis sexo, idade, estado civil, convivência familiar, escolaridade, local e apropriação de moradia, renda familiar mensal e participação na vida econômica familiar. Os resultados são demonstrados através de números absolutos (n) e em percentuais (\%). 
Tabela 1 - Caracterização sociodemográfica e econômica das pessoas com DI de um município de pequeno porte do interior do RS, $2015(n=77)$.

\begin{tabular}{|c|c|c|c|}
\hline Variáveis & Condição & $\mathbf{n}$ & $\%$ \\
\hline \multirow[t]{2}{*}{ Sexo } & Masculino & 46 & 59,74 \\
\hline & Feminino & 31 & 40,25 \\
\hline \multirow{5}{*}{ Idade } & Terceira Infância (6 aos 11 anos) & 06 & 7,79 \\
\hline & Adolescência (12 aos 20 anos) & 14 & 18,18 \\
\hline & Vida adulta (21 aos 40 anos) & 30 & 38,96 \\
\hline & Vida adulta intermediária (41 aos 65 anos) & 23 & 29,87 \\
\hline & Vida adulta tardia (dos 65 anos em diante) & 04 & 5,19 \\
\hline \multirow{3}{*}{ Estado civil } & Solteiros & 71 & 92,20 \\
\hline & Casado & 05 & 6,49 \\
\hline & Separado & 01 & 1,29 \\
\hline \multirow{8}{*}{ Convivência familiar } & Pais & 23 & 29,87 \\
\hline & Mãe & 22 & 28,57 \\
\hline & Irmão/irmã & 09 & 11,68 \\
\hline & Outros & 08 & 10,38 \\
\hline & Cônjuge/companheiro & 07 & 9,09 \\
\hline & Tio/tia & 03 & 3,89 \\
\hline & Filho & 01 & 1,29 \\
\hline & Sozinho & 01 & 1,29 \\
\hline \multirow{2}{*}{ Escolaridade } & Analfabetos & 43 & 55,84 \\
\hline & Ensino Fundamental Incompleto & 34 & 44,15 \\
\hline \multirow{3}{*}{ Local de moradia } & Periferia & 36 & 46,75 \\
\hline & Central & 25 & 32,46 \\
\hline & Rural & 12 & 15,58 \\
\hline \multirow{3}{*}{ Apropriação de moradia } & Própria & 58 & 75,32 \\
\hline & Aluguel & 11 & 14,28 \\
\hline & Outra & 08 & 10,38 \\
\hline \multirow{5}{*}{ Renda familiar mensal } & 1 a 2 salários mínimos & 43 & 55,84 \\
\hline & 2 a 5 salários mínimos & 25 & 32,46 \\
\hline & Variável & 04 & 5,19 \\
\hline & Não declarou & 04 & 5,19 \\
\hline & Menos de um salário mínimo & 01 & 1,29 \\
\hline \multirow{4}{*}{$\begin{array}{l}\text { Participação na vida } \\
\text { econômica familiar }\end{array}$} & Benefício de Prestação Continuada (BPC) & 57 & 74,02 \\
\hline & Sustento familiar & 18 & 23,37 \\
\hline & Bolsa família & 01 & 1,29 \\
\hline & Outra & 01 & 1,29 \\
\hline
\end{tabular}

Fonte: Dados da pesquisa (2015).

Quanto ao diagnóstico e acompanhamento clínico-terapêutico e educacional, apresentam-se na Tabela 2, os diagnósticos encontrados, conforme o CID- 10 (F70, F71, F72, F73, F79, F84, G80 e Q90), em ordem decrescente, a identificação dos profissionais que os forneceram e dos acompanhamentos clínico-terapêutico e educacional que os sujeitos recebiam por ocasião da coleta de dados. 
Tabela 2 - Diagnóstico e acompanhamento clínico-terapêutico e educacional de pessoas com DI, município do interior do RS, $2015(n=77)$.

\begin{tabular}{|c|c|c|c|}
\hline Variáveis & Condição & $\mathbf{n}$ & $\%$ \\
\hline \multirow{8}{*}{ Diagnóstico } & F79 (Retardo Mental Não Especificado) & 31 & 40,25 \\
\hline & F70 (Retardo Mental Leve) & 11 & 14,28 \\
\hline & F71 (Retardo Mental Moderado) & 09 & 11,68 \\
\hline & F72 (Retardo Mental Grave) & 08 & 10,38 \\
\hline & Q90 (Síndrome de Down) & 08 & 10,38 \\
\hline & G80 (Paralisia Cerebral) & 06 & 7,79 \\
\hline & F84 (Autismo) & 03 & 3,89 \\
\hline & F73 (Retardo Mental Profundo) & 01 & 1,29 \\
\hline \multirow{4}{*}{$\begin{array}{l}\text { Profissional que forneceu o } \\
\text { diagnóstico) }\end{array}$} & Médico Neurologista & 39 & 50,64 \\
\hline & Médico Psiquiatra & 24 & 31,16 \\
\hline & Médico Pediatra & 07 & 9,09 \\
\hline & Médico Clínico Geral & 07 & 9,09 \\
\hline \multirow{5}{*}{$\begin{array}{c}\text { Tipo de } \\
\text { acompanhamento clínico }\end{array}$} & Médico Psiquiatra & 35 & 45,45 \\
\hline & Médico Clínico Geral & 34 & 44,15 \\
\hline & Médico Neurologista & 20 & 25,97 \\
\hline & Médico Pediatra & 06 & 7,79 \\
\hline & Nenhum & 02 & 2,59 \\
\hline \multirow{5}{*}{$\begin{array}{c}\text { Tipo de acompanhamento } \\
\text { terapêutico }\end{array}$} & Psicólogo & 27 & 35,06 \\
\hline & Terapeuta Ocupacional & 21 & 27,27 \\
\hline & Fonoaudiólogo & 17 & 22,07 \\
\hline & Fisioterapeuta & 10 & 12,98 \\
\hline & Nenhum & 18 & 23,37 \\
\hline $\begin{array}{c}\text { Tipo de acompanhamento } \\
\text { educacional }\end{array}$ & Educação Especial & 36 & 46,75 \\
\hline
\end{tabular}

Fonte: Dados da pesquisa (2015).

Na Tabela 3, apresenta-se a caracterização da saúde atual de pessoas com DI na ocasião da coleta de dados da pesquisa. 
Tabela 3 - Caracterização da saúde atual de pessoas com DI, município do interior do RS, 2015 (n=77).

\begin{tabular}{|c|c|c|c|}
\hline Variáveis & Condição & $\mathbf{n}$ & $\%$ \\
\hline \multirow{2}{*}{ Visão } & Sem particularidades & 55 & 71,42 \\
\hline & Com déficit & 22 & 28,57 \\
\hline \multirow{2}{*}{ Audição } & Sem particularidades & 68 & 88,31 \\
\hline & Com déficit & 09 & 11,68 \\
\hline \multirow{3}{*}{ Polifarmácia } & $\begin{array}{ll}\text { Dois } \quad \text { a } & \text { três } \\
\text { medicamentos/dia } & \end{array}$ & 23 & 29,87 \\
\hline & $\begin{array}{l}\text { Mais de quatro } \\
\text { medicamentos/dia }\end{array}$ & 23 & 29,87 \\
\hline & Não usa medicamentos & 19 & 24,67 \\
\hline \multirow{13}{*}{$\begin{array}{c}\text { Principais } \\
\text { medicamentos usados }\end{array}$} & Carbamazepina $2 \%$ & 24 & 31,16 \\
\hline & Biperideno & 16 & 20,77 \\
\hline & Gardenal & 10 & 12,98 \\
\hline & Haloperidol & 09 & 11,68 \\
\hline & Depakene & 08 & 10,38 \\
\hline & Risperidona & 09 & 11,68 \\
\hline & Puran T4 & 09 & 11,68 \\
\hline & Rivotril & 11 & 14,28 \\
\hline & Haldol & 08 & 10,38 \\
\hline & Clorpromazina & 05 & 6,49 \\
\hline & Fluoxetina & 05 & 6,49 \\
\hline & Sivastatina & 06 & 7,79 \\
\hline & Hidroclorotiazida & 04 & 5,19 \\
\hline \multirow{3}{*}{ Fumo } & Não & 66 & 85,71 \\
\hline & Sim & 08 & 10,38 \\
\hline & Ex-fumante & 03 & 3,89 \\
\hline \multirow{3}{*}{ Álcool } & Não & 73 & 94,80 \\
\hline & Sim & 03 & 3,89 \\
\hline & Parou há um ano & 01 & 1,29 \\
\hline
\end{tabular}

Fonte: Dados da pesquisa (2015).

\section{Discussão}

Os dados levantados neste estudo nos permitiram retratar quem são as pessoas com DI, residentes em um município de pequeno porte do interior do RS. Tal conhecimento poderá beneficiar a implementação de programas e ações que permitam atender às demandas sociais, melhorando a qualidade de vida dessa população.

Acerca dos dados sociodemográficos de pessoas com DI, note-se que houve prevalência do sexo masculino sobre o feminino. A literatura científica nacional que associa as variáveis sexo e DI é ainda escassa; exceto os dados gerais do IBGE que mostram 0,8\% da população brasileira apresenta DI, sendo 0,9\% homens e 0,7\% mulheres (Ibge, 2010). Dados internacionais do início dos anos 90, apresentavam a prevalência de homens com DI - razão de 4:3 (Assunção Jr, 1994). No que tange a faixa etária, o estudo de Assunção Jr (1994) não fazia referências claras, no entanto, o autor alertava que dados referentes a esta devem ser considerados, levando em conta também as características da região e do meio socioeconômico estudados. Os dados deste estudo, atual e regional, informaram a razão de 10:7; portanto, os dados diferem ligeiramente.

No que tange ao estado civil, a maioria são solteiros. Estes achados conferem com outro estudo cuja a explicação são, por exemplo, o isolamento domiciliar, a dependência nas atividades de vida diária e a falta de autonomia da pessoa com DI para gerir a vida afetiva e manter um relacionamento conjugal (Aoki \& Oliver, 2013).

Quanto ao cuidado à pessoa com DI, averiguou-se que era realizado quase predominantemente por cuidadores que apresentavam algum grau de parentesco. De acordo Sena e Gonçalves (2008), no Brasil e no mundo, há uma tendência de que os cuidados de um familiar com deficiência ou com limitações fisiológicas próprias do envelhecimento sejam conduzidos pela própria família, ou seja, há uma naturalização da ideia de família cuidadora. 
Os quase 10\% de idosos que não convivem com seus familiares encontram-se na única ILPI do município, de natureza filantrópica, não governamental e sem fins lucrativos. Ressalta-se que as ILPI têm como objetivos a socialização, a reintegração, a estimulação e o apoio aos idosos, com atendimento integral nas áreas de saúde, higiene, alimentação e habitação, ou seja, pode ser compreendida como uma residência com condições de acolher idosos em situação de carência de renda ou familiar, com dificuldades de realizar tarefas diárias e que necessitam de cuidados prolongados (Brasil, 2005). Portanto, os dados relativos à convivência das pessoas com DI deste estudo, confirmam dados da literatura, encontram-se em convívio familiar e/ou em ILPI.

Outro fator alarmante encontrado refere-se à baixa taxa de escolaridade observada nesta população, o que pode ser um reflexo da dificuldade de aplicação da lei que prevê garantia de educação às pessoas com deficiência em todos os níveis de ensino. De fato, o direito à educação garante a participação do aluno com deficiência no ambiente escolar. Logo, a inclusão educacional é a garantia de acesso e permanência em um ensino de qualidade, porém, por mais que o direito à educação esteja formalizado constitucionalmente, há muito que intervir para que as práticas escolares se aproximem mais das diretrizes da educação inclusiva (Guerreiro, 2012). Neste contexto, a educação tem sido identificada como um dos fatores mais importantes que influenciam a inclusão social (Burchardt, 2005). Deste modo, os dados encontrados caracterizam um problema de ordem pública, tornando-se necessárias a consolidação das políticas de incentivo a escolaridade das pessoas com DI deste município. Notadamente, maiores níveis de escolaridade e qualificação profissional estão associados com maiores chances de ingresso no mercado de trabalho. Desta forma, a educação caracteriza-se como um instrumento de inclusão nas atividades laborais (Dorval, 2006).

No que tange ao local de moradia, constatou-se que quase metade reside na periferia da cidade e possuía casa própria, fato que amplia a visibilidade da política nacional de habitação, que beneficia as pessoas de baixa renda. Dados sobre condições de habitação da população são fundamentais para o estabelecimento de ações que visem à promoção da qualidade de vida dos indivíduos, das famílias e da comunidade (Opas, 1998). Os dados deste estudo, possibilitam dizer que a condição de moradia de pessoas com DI é favorável.

No que se refere à renda familiar mensal, dos que conviviam com seus familiares, pouco mais da metade recebiam de um a dois salários mínimos e pouco mais de um terço de dois a cinco salários mínimos; entende-se que essa condição econômica limita o acesso a bens de serviço e de consumo, como alimentação, educação, lazer e moradia, entre outros aspectos da saúde. De acordo com Oliveira et al. (2008) as dificuldades financeiras interferem no cuidado de pessoas com DI, pois a renda mensal baixa não supre as necessidades básicas, como por exemplo medicamentos, cuidados especializados em saúde e educação e outras básicas como alimentação, transporte e lazer. Assim, constata-se que o baixo nível socioeconômico das famílias produz privações, como por exemplo - isolamento social e a violência no cotidiano. Assim, políticas públicas voltadas para a inclusão das pessoas com DI devem manter foco nas questões que permeiam a participação e a inclusão social, a partir de ações educativas e assistenciais, considerando o sujeito em sua singularidade, complexidade, integralidade e na inserção sociocultural.

Foi encontrado um elevado número de pessoas (maioria) que recebem benefícios previdenciários e sociais. Segundo a Lei $\mathrm{n}^{\circ}$ 8.742, de 7 de dezembro de 1993, o Benefício de Prestação Continuada - BPC - garante um salário mínimo mensal à pessoa portadora de deficiência que comprove não possuir meios de prover a própria manutenção e nem de tê-la provida por sua família (Brasil, 1993). Segundo o estudo de Santos (2011), realizado junto à população similar, muitas vezes, o BPC e as aposentadorias por invalidez são a única fonte de renda de toda uma família (Santos, 2011).

Convém ressaltar que foi constatado, que em algumas famílias as pessoas com DI exerciam alguma atividade rentável (apesar de esporádica), por exemplo, jardinagem. No entanto, os familiares/cuidadores descartaram a ideia de inseri-lo no mercado de trabalho, com receio de que houvesse a suspensão do BPC, mesmo tendo conhecimento da alteração na legislação, 
que garante a retomada do recebimento do benefício, caso o sujeito perca o emprego (Brasil, 2011). Pode-se dizer que este dado indica uma falta de reconhecimento do familiar/cuidador das condições das pessoas com DI colocar-se como cidadão produtivo e autônomo, com potencial para desenvolvimento de habilidades ocupacionais e sociais para além da família e, ainda, reforça a manutenção da vulnerabilidade dessas famílias, uma vez que, os rendimentos de fontes como o BPC tendem a serem inferiores aos de uma atividade laboral como a de jardinagem.

A codificação das doenças no Brasil segue, em geral, aquela definida pela Classificação Internacional de Doenças e Problemas de Saúde (CID-10) (OMS, 1993), que utiliza o termo retardo mental e o classifica desde retardo mental leve (F70) até retardo mental profundo (F73), outro retardo mental (F78) e retardo mental não-especificado (F79), sendo os escores de quociente de inteligência (QI) um dos principais quesitos.

Quanto aos resultados sobre o diagnóstico de pessoas com DI deste estudo, obteve-se a prevalência do diagnóstico de "Retardo Mental não especificado" (F79), usado quando não se pode realizar avaliações por meio dos instrumentos habituais/psicométricos de inteligência. Assim, refere-se uma suposição de "retardo mental" (termo ainda usado na área da Saúde e da Educação) e, por isso, usado genericamente nos atestados de DI. Destaca-se, que o termo DI veio justamente substituir e ampliar a conotação e representação de termos anteriores tais como "débil mental", "idiota", "retardado mental", "excepcional", "incapaz mentalmente" e erroneamente associados a transtornos mentais, como o "maluco" ou o "louco", construídos e utilizados por médicos da corrente organicista, em determinados períodos históricos da sociedade europeia (Pessotti, 1984).

Constatou-se ainda, que tais diagnósticos foram elaborados por diferentes especialidades da Medicina (neurologia, psiquiatria, clínica geral e pediatria), prevalecendo os estabelecidos por neurologistas e psiquiatras. A melhor forma de definir a DI é por uma visão multidimensional, de acordo com a qual a DI seria uma incapacidade caracterizada por limitação significativa no funcionamento intelectual e no comportamento adaptativo expresso nas habilidades conceituais, sociais e práticas (Ammr, 2006).

Convém discutir o aparecimento dos diagnósticos F84 (Transtorno do Espectro Autista), G80 (Paralisia Cerebral PC) e Q90 (Síndrome de Down). A propósito, esses podem estar na base da DI e, por isso, substitutivos dos diagnósticos de DI. A síndrome de Down, por exemplo, integra o capítulo das malformações congênitas, e a literatura refere a possibilidade de haver diferentes níveis de intelectualidade, podendo ser leve (QI50-70), moderado (QI 35-50) e, raramente, grave (QI 20-35) (Oms, 1993; Brasil, 2013). É importante salientar que os atestados médicos, encontrados neste estudo, muitas vezes, apresentavam além dos referidos CID declarações como "Retardo Mental" ou "Atraso Mental".

Outra discussão pertinente acerca deste tema (diagnóstico) é a de poder ser interpretado como uma determinação incontestável - um "rótulo"; enquanto se sabe que os diagnósticos devem fornecer informações necessárias para os processos de intervenção. No caso de um diagnóstico de DI, é importante que o profissional esteja ciente da complexidade desse momento para a família e, por isso, convém que a avaliação e o diagnóstico sejam fechados multiprofissionalmente, envolvendo diversas especialidades, como: fonoaudiólogos, educadores especiais, terapeutas ocupacionais, entre outros. Deve haver comunicação clara entre os envolvidos para que o diagnóstico seja fidedigno, assim como ofertado de um modo humanizado, e indicativo das possibilidades de acompanhamentos terapêuticos e educacionais. A este respeito, discutem-se, a seguir, as condições dos sujeitos desta pesquisa quanto aos cuidados longitudinais recebidos.

$\mathrm{Na}$ Tabela 2, pode-se evidenciar que quase a totalidade dos sujeitos deste estudo recebem acompanhamento médico em diversas especialidades (clínica geral; psiquiatria; neurologia; pediatria), com regularidade anual. Segundo o Ministério da Saúde (Brasil, 2006), além dos acometimentos intelectuais, as pessoas com DI apresentam outros problemas de saúde, necessitando, assim, de atendimentos em diversas especialidade médicas, como constatado neste estudo. Convém destacar que o número elevado de atendimentos médicos, possa ser devido à cidade ser de pequeno porte e apresentar 84,79\% de cobertura 
em Estratégia de Saúde da Família (ESF) e 91,86\% de cobertura com Agentes Comunitários de Saúde (ACS), ou seja, conta com uma Atenção Básica abrangente e referenciada (as especialidades médicas são ofertadas em hospital regional).

No entanto, não há na rede pública de saúde um ambulatório especializado em DI. Famílias com poder aquisitivo baixo contam com uma instituição - não governamental e filantrópica - que realiza simultaneamente o atendimento terapêutico e educacional. Famílias com maior poder aquisitivo procuram por acompanhamento terapêutico em Fisioterapia, Fonoaudiologia, Psicologia, Terapia Ocupacional, entre outros, em cidades vizinhas com maior disponibilidade desses profissionais.

Os dados deste estudo mostraram que o município ainda carece de uma rede apropriada de atenção à pessoa com DI; os acompanhamentos existentes revelam o empenho dos familiares e responsáveis que têm recursos financeiros para saírem da cidade, enquanto que os menos favorecidos financeiramente usam os serviços públicos e filantrópicos disponíveis. A atenção integral à saúde, conforme prevista em legislação, compreende ações de promoção, prevenção, assistência, reabilitação e manutenção da saúde; inclusive a garantia de internação hospitalar ou cuidado domiciliar (Brasil, 2006). Tais exigências são praticamente impossíveis de serem cumpridas em municípios de pequeno porte e com restrição orçamentária.

A situação se agrava ainda mais, no caso de pessoas com DI envelhecidas, que tal como as outras podem apresentar agravos de saúde. De acordo com Rimmer (2002), as condições na saúde durante o curso de vida não são diferentes para uma pessoa com DI. Aqueles que praticam bons hábitos de saúde, alimentando-se, exercitando-se, mantendo uma avaliação médica regular, prevenindo doenças e mantendo adequado peso corporal, podem ser considerados com um alto nível de saúde continuada. Considera-se que o cuidado e pesquisas sobre o funcionamento dos sistemas invisíveis/pouco aparentes (por exemplo, o endócrino e circulatório) impõem-se como uma necessidade para os profissionais que se envolvem efetivamente na atenção à saúde de pessoas com DI, podendo, assim, favorecer intervenções clínico-terapêuticas abrangentes que visam responder aos princípios da integralidade e longitudinalidade do cuidado em saúde dessa população.

As ações devem ser multiprofissionais, interdisciplinares e intersetoriais, para que sejam possíveis respostas mais efetivas às necessidades de saúde das pessoas. Cecílio (2006) afirma que deve haver uma articulação entre as diversas áreas, por meio de ações integrais. A integralidade é princípio norteador para a apreensão das necessidades dos sujeitos atendidos, bem como para a compreensão e formulação das respostas a serem dadas (Ayres, 2009), incluindo aspectos muito importantes: as necessidades não se resumem à doença; a prevenção e a assistência devem estar articuladas, os problemas são complexos e envolvem o contexto sociocultural, o usuário é um sujeito (com história, valores, desejos).

Quanto à caracterização da saúde atual de pessoas com DI na ocasião da coleta de dados desta pesquisa, os aspectos sensoriais, principalmente visão e audição, este estudo revelou que a maioria não apresentava comprometimentos visuais e nem auditivos. Talvez, os índices desses déficits ainda estejam baixos devido ao fato de a mediana de idade ser 33 anos (seis anos de idade o mais novo e 74 anos o mais velho), ou seja, a maioria da população deste estudo ainda não é considerada idosa.

Ainda na linha do reconhecimento das condições de saúde das pessoas com DI, este estudo revelou um grande uso de medicamentos, principalmente os chamados psicotrópicos, utilizados para controlar distúrbios comportamentais, além de crises epiléticas (Rang et al., 2003).

Segundo Nottestad e Linaker (2003), poucos medicamentos são prescritos para sintomas específicos, sendo muitos administrados apenas para impedir aberrações comportamentais ou ainda, para inibir crises epilépticas, as quais estão constantemente associadas às neuropatologias. Para que estes medicamentos sejam verdadeiramente benéficos, sua discriminação e dosagem devem ser controladas, pois, uma administração incorreta pode ser prejudicial. Por exemplo, os anticonvulsivantes podem ter efeito danoso dependendo da dose, podendo interferir em funções motoras, intelectuais e provocar sedação. 
Logo, os cuidados que se devem ter na administração de medicamentos a pessoas com DI exigem conscientização e capacitação daqueles que a fizerem, sejam eles familiares, profissionais da área de saúde ou de instituições especializadas.

Um outro aspecto que deve ser levado em consideração é o alto custo de alguns medicamentos utilizados no tratamento de neuropatologias. Por este motivo, muitas famílias, incapacitadas de adquirir estes fármacos devido à baixa renda, acabam interrompendo o tratamento causando ainda mais sofrimento a pessoa com DI.

Em relação aos hábitos de tabagismo e etilismo é interessante observar o alto percentual de sujeitos que nunca fumaram e que nunca beberam. Hábitos nocivos praticamente inexistentes, possivelmente pela concepção protecionista de "eternas crianças" e pela forte influência da família nos cuidados em saúde desses sujeitos que impedem o primeiro contato com essas substâncias, evitando assim, que pessoas com DI adquiram esses hábitos nocivos à sua saúde.

Os dados encontrados, mesmos que comparados com outros estudos, se limitam a uma realidade local do grupo estudado, pois se trata de um recorte que reflete características específicas dessa população, necessitando assim de mais pesquisas para fomentar um maior levantamento das particularidades e dificuldades enfrentadas por pessoas com DI.

\section{Conclusão}

Com a realização deste estudo, foi possível conhecer quem são as pessoas com DI de uma cidade de pequeno porte, do interior do Rio Grande do Sul. Ao caracterizar os participantes deste estudo (pessoas com DI), foi possível constatar que, em muitos aspectos, suas condições de vida e saúde assemelham-se à população em geral e, em outros, diferenciam-se. Com relação às semelhanças, constatou-se a presença de doenças crônico-degenerativas e a polifarmácia (uso de diversos medicamentos). Os recursos financeiros para a sobrevivência são limitados, a maioria depende do BPC ou de aposentadoria (por idade) e, geralmente, são oriundos de classes sociais menos favorecidas.

No que tange à escolarização constatou-se que o a maioria de pessoas com DI são analfabetas ou frequentaram no máximo cinco anos de escolarização contrariando a lei de implantação da escola inclusiva. Vale salientar que frequentar a escola é essencial para essas pessoas aprenderem, não apenas habilidades acadêmicas, mas também para desenvolverem habilidades e potencialidades.

Quanto às condições de vida/saúde das pessoas com DI, deste estudo pode-se dizer que são satisfatórias. Apesar de pertencentes a um município de pequeno porte, tais dados poderão colaborar com os profissionais e gestores da Saúde e da Educação, subsidiando assim discussões voltadas para o aprimoramento do cuidado longitudinal necessário junto a pessoas com DI.

Ressalta-se que os achados do presente estudo se limitam a uma realidade específica. Assim, sugere-se a realização de futuras investigações desta natureza, em novos cenários, no intuito de se alcançar uma melhor compreensão das condições de vida e saúde da pessoa com DI no contexto de comunidade.

\section{Referências}

AMMR (2006). American Association on Mental Retardation. Retardo mental: definição, classificação e sistemas de apoio. (10a ed.), Artmed.

Aoki, M., \& Oliver, F. C. (2013). Pessoas com deficiência moradoras de bairro periférico da cidade de São Paulo: estudo de suas necessidades. Cadernos de Terapia Ocupacional da UFSCar, São Carlos, 21 (2), 391-398.

APA (2014). American Psychiatric Association. Manual diagnóstico e estatístico de transtornos mentais - DSM-5. Editora Artmed.

Assumpção Jr, F (2004). Psiquiatria da infância e da adolescência. Santos.

Ayres, J. R. C. M. (2009). Organização das ações de atenção à saúde: modelos e práticas. Saúde Soc.,18 (supl. 2 ), 11-23.

Brasil (1988). Constituição da República Federativa do Brasil: promulgada em 5 de outubro de 1988. Brasília. 
Brasil (2005). Subsecretaria de Direitos Humanos. Plano de Ação para o Enfrentamento da Violência Contra a Pessoa Idosa. Brasília: Subsecretaria de Direitos Humanos.

Brasil (2006). Ministério da Saúde. Secretaria de Atenção à Saúde. Departamento de Ações Programáticas Estratégicas. Manual de legislação em saúde da pessoa com deficiência. Brasília: Editora do Ministério da Saúde.

Brasil (2011). Lei no 12.435 de 06 de julho de 2011. Altera a Lei n. 8.742, de 7 de dezembro de 1993, que dispõe sobre a organização da Assistência Social. Dispõe sobre a organização do setor e institui o Sistema Único de Assistência Social (SUAS). Diário Oficial [da] República Federativa do Brasil, Poder Executivo, Brasília, DF.

Brasil (2012). Conselho Nacional de Saúde. Resolução n 466, 2012. Diretrizes e Normas regulamentadoras de pesquisa envolvendo seres humanos. Brasília. Seção 1 p. 59.

Brasil (2013). Ministério da Saúde. Secretaria de Atenção à Saúde. Departamento de Ações Programáticas Estratégicas. Diretrizes de atenção à pessoa com Síndrome de Down. Brasília: Ministério da Saúde.

Burchardt, T. (2005). The education and employment of disabled young people: Frustrated ambition. London School of Economics and Political Science.

Cecilio, L. C. O. (2006). As necessidades de saúde como conceito estruturante na luta pela integralidade e equidade na atenção em saúde. In: PINHEIRO, R.; MATTOS, R.A. (Org.). Os sentidos da integralidade na atenção e no cuidado à saúde. 6.ed. Rio de Janeiro: UERJ, IMS/Abrasco: $115-28$.

Doval, J. L. M. (2006). Inclusão de pessoas portadoras de deficiência no mercado de trabalho: desafios e tendências. Dissertação de mestrado. Universidade Federal do Rio Grande do Sul. Porto Alegre, RS.

Girardi, M. et al. (2012). O envelhecimento em deficientes intelectuais. RBCEH. 9(1), 79-89.

Guerreiro, E. M. B. R. (2012). A acessibilidade e a educação: um direito constitucional como base para um direito social da pessoa com deficiência. Revista Educação Especial, 25 (43), 217-32.

IBGE (2017). Instituto Brasileiro de Geografia e Estatística. Censo demográfico 2010. http://www.ibge.gov.br/home/estatistica/populacao/censo2010/ (acessado em 06/Fev/2017).

Kassar, M. C. M. (2012). Educação Especial no Brasil: Desigualdades e Desafios no Reconhecimento da Diversidade. Educ. Soc., Campinas, 33 (120), 833 849.

Mantoan, M. T. E. (2015). Inclusão escolar: O que é? Por quê? Como fazer? Summus.

Nottestad, J. \& Linaker, O. M. (2003). Psychotropic drug use among people with intellectual disability before and after deinstitucionalization. Journal of Intellectual Disability Research, Oxford, 47, 464-471.

O’leary, L. et al. (2018). Early death and causes of death of people with intellectual disabilities: a systematic review. J. Appl. Res. Intellect Disabil. 31 , 325-42.

Oliveira, M. F. S. et al. (2008). Qualidade de vida do cuidador de crianças com paralisia cerebral. RBPS, Fortaleza, 21 (4), $275-280$.

OMS (1993). Organização Mundial da Saúde. Classificação de transtornos mentais e de comportamento da CID-10 - Descrições clínicas e diretrizes diagnósticas. Porto Alegre: Artes Médicas.

OPAS (1998). Organização Panamericana de Saúde. La Saluden las Américas. Washington.

Pereira, A. S. et al. (2018). Metodologia da Pesquisa. RS: UFSM, NTE.

Pessotti, I. (1984). Deficiência mental: da superstição à ciência. Edusp.

Rang, H. P. et al. (2003). Farmacologia. (5a ed.), Ed. Elsevier.

Rimmer, J. H. (2015). Health promotion for people with disabilities: the emerging paradigm shift from disability prevention to prevention of secondary conditions. <http://www.ncpad.org/wthpprs/healthpropwdshift.htm>.

Santos, W. R. (2011). Deficiência e BPC: o que muda na vida das pessoas atendidas. Ciência \& Saúde Coletiva. 16 (1), $787-796$.

Sena, E. L. S. \& Goncalves, L. H. T. (2008). Vivências de familiares cuidadores de pessoas idosas com doença de Alzheimer - Perspectiva da filosofia de Merleau Ponty. Texto \&Contexto Enferm. 17(2), 232-240.

Schalock, R. L. et al. (2010). Intellectual disability: definition, classification, and systems of supports. (11a ed.), Washington DC: American Association on Intellectual and Developmental Disabilities. 